\title{
Psychopathology in Sri Lankan patients with first-episode psychosis
}

\author{
NS Balasooriya, HGVW Wijesiri, R Hanwella, V De Silva
}

DOI: http://doi.org/10.4038/sljpsyc.v8i1.8135

\section{Abstract \\ Background}

First episode psychosis (FEP) is the first episode of a psychiatric illness with psychotic symptoms. Delay in diagnosis increases the duration of untreated psychosis (DUP) and leads to poorer outcome.

Aims

To explore the psychopathology of patients with FEP presenting to the National Hospital of Sri Lanka (NHSL).

\section{Methods}

This was an observational descriptive study. Patients admitted to ward-59, NHSL from March 2012 to November 2013 who met inclusion criteria were considered for the study. Data was collected retrospectively from bed head tickets using a structured questionnaire.

\section{Results}

The sample size was 101, of which 57 were male. A majority of the participants were in the 13-26 year age group. Delusions were the most common psychopathology noted in this sample, with persecutory themes being the most common content. Of the hallucinations, second person auditory hallucinations were the commonest type. Non-euthymic mood was seen in over two-thirds of participants, with depressed mood being the most common finding. Insight was poor in over half the participants. Schizophrenia was the most common diagnosis noted in this group of patients, followed by mania with psychotic features.

\section{Conclusions}

Persecutory delusions, second person auditory hallucinations, mood and speech abnormalities were prominent among participants of this study. A better understanding of psychopathology in FEP may help to reduce DUP.

Key words: First episode psychosis, psychopathology, Sri Lanka

SL J Psychiatry 2017; 8(1): 21-25

\section{Introduction}

First episode psychosis (FEP) refers to the first episode of a psychiatric illness which presents with psychotic symptoms. Identification of the first episode can be difficult and delayed especially if the onset is insidious. Early identification of the first episode is of crucial importance as early interventions lead to better out comes. Psychopathology during the first episode can be variable and often deviates from the characteristic descriptions of more established illness.

The onset of FEP often affects young people in their teens or in their 20s (1-3). Hafner et al. in their study of 276 first-admitted patients with schizophrenia aged 12-59 years, described a predominance of persecutory delusions in older patients, compared with younger patients who were reported to have more non-specific psychotic symptoms (4). This suggests that the phenomenology in young first episode patient may differ from those with more established illness.

Studies in patients with established schizophrenia suggest that themes of delusional beliefs are related to themes of life problems and goals $(5,6)$. As life issues of younger patients differ from those of older patients a difference in themes of delusions and hallucinations can be expected.

The duration of untreated psychosis (DUP) is defined as the period between onset of symptoms of psychosis and the start of antipsychotic treatment (7). A long DUP is associated with a poor treatment outcomes (8). Lack of awareness about the phenomenology of the first episode may lead to delay in identification of the disorder and a long DUP.

Published studies that have explored the phenomenology of the first episode patient are scarce. In one study 
done in Melbourn, Australia, delusions of persecution, reference and second person auditory hallucinations were the most commonly reported psychotic symptoms at initial presentation (9). There have been no published studies done in Sri Lanka exploring the phenomenology of the first episode patient.

A better awareness of the phenomenology of the firstepisode patient may aid early intervention, which would lead to better long term outcomes. Therefore the aim of this research was to study the psychopathology of patients with first episode psychosis admitted to the University Psychiatry Unit of the National Hospital of Sri Lanka.

\section{Method}

This was an observational descriptive study, carried out at the University Psychiatry unit of the National Hospital of Sri Lanka. The National Hospital of Sri Lanka is the largest teaching hospital in Sri Lanka, consisting of 3000 beds. The University Psychiatry Unit at the National Hospital provides inpatient care for acute patients, outpatient care for day patients and liaison services to the other units. The data was collected retrospectively, using data recorded in the bed head tickets (BHT's).

Ethical clearance was obtained from the Ethics Review Committee of the National Hospital of Sri Lanka. During data collection, anonymity was maintained and patient name and contact details were not extracted.

Patients who presented with a first episode of psychosis, defined as a first episode of psychiatric illness with psychotic symptoms, were considered eligible to be included in this study. Convenience sampling was used to select the bed head tickets of eligible patients. All patients admitted to the University Psychiatry Unit, National Hospital of Sri Lanka for the first time from 01.03.2012 to 30.11.2013 were included provided they had delusions and hallucinations during the admission or carried a diagnosis of drug-induced psychosis (ICD category -F1x.5), schizophrenia (ICD cat: -F20), schizophreniform disorder (ICD cat: -F20.8), schizoaffective disorder (ICD cat: -F25), delusional disorder (ICD cat: -F22), acute and transient psychotic disorder (ICD cat: -F23), severe depressive episode with psychotic symptoms (ICD cat: -F32.3), bipolar affective disorder current episode severe depression with psychotic features (ICD cat: -F31.5), or bipolar affective disorder current episode mania with psychotic features (ICD cat: -F31.2). If patients had received treatment prior to the admission as an outpatient or inpatient to NHSL with the duration of antipsychotic use being less than 12 weeks (of continuous use) they were considered eligible to be included in the study (10).
Patients who did not have psychotic symptoms or who did not have the above diagnoses, and those who had received more than 12 weeks of continuous antipsychotic treatment prior to the admission as an inpatient or an outpatient, were excluded from the study (10).

A structured questionnaire was used to extract data from the bed head tickets. Data collection was done by doctors with post-MD training in psychiatry, who had adequate knowledge of psychopathology and phenomenology to extract the data from the bed head tickets. Prior to starting the data collection, those collecting data participated in workshops, which provided training in the use of the structured questionnaire for extraction of relevant psychopathology. Data regarding demographic details, sources of referrals, duration of untreated psychosis, psychoactive substance use, attempted suicides, family history of psychiatric illnesses, abnormalities in the appearance and behaviour, speech, mood, thoughts, perceptions, cognitive functions, insight, and omissions in recording psychopathology in the bed head tickets were collected.

Descriptive statistics in the form of means (standard deviations) and percentages (counts) were computed using the Statistical Package for the Social Sciences (SPSS, SPSS Inc., Chicago, IL, USA) and Microsoft Excel.

\section{Results}

The total number of patients with FEP admitted to the University Psychiatry Unit of the National Hospital of Sri Lanka during the study period was 101, of whom 57 were male. Of the participants, 49 were between 13-26 years, 42 between 27-60 years, and 10 were older than 60 years (Table 1). Catatonia was present in 5 (4.9\%) of participants, speech abnormalities in 43 (42.5\%), thought disorder in 13 (12.87\%), and non-euthymic mood in 74 (72.3\%), of which depressed mood was more common. Mood incongruity was present in 19 (18.8\%) and suicidal ideas in 10 (9.9\%).

Delusions were the most common psychopathology noted among the study participants. Delusions were present in $88(87.1 \%)$ of participants, of which the commonest themes were persecutory (64.77\%), reference (38.63\%), thought alienation (22.7\%), and grandiose (19.3\%). Hallucinations were present in 67 (66.3\%) participants, of which auditory hallucinations were seen most commonly (second person $46.26 \%$, third person $40.29 \%$, and command $13.43 \%$ ). Tactile hallucinations were present in 12 (17.9\%), and olfactory hallucinations in 4 (5.97\%). Insight was poor in 57 (56.43\%) participants, and partial in 23 (22.77\%). The more commonly seen diagnoses were schizophrenia in 51 (50.49\%) participants, mania with psychotic features in 13 (12.87\%), delusional disorder in 9 (8.91\%), acute and transient psychotic disorder in 9 (8.91\%), and psychotic depression in 7 (6.93\%). 


\begin{tabular}{|c|c|c|}
\hline Psychopathology & Number of patients & Percentage \% \\
\hline Catatonia & 5 & 4.9 \\
\hline Speech abnormalities & 43 & 42.5 \\
\hline Thought disorder & 13 & 12.9 \\
\hline Non-euthymic mood & 74 & 72.3 \\
\hline Mood incongruity & 19 & 18.8 \\
\hline Suicidal ideas & 10 & 9.9 \\
\hline Delusions & 88 & 87.1 \\
\hline Hallucinations & 67 & 66.3 \\
\hline Poor insight & 57 & 56.4 \\
\hline Partial insight & 23 & 22.8 \\
\hline Delusions - themes: & Number of Patients & Percentage \% \\
\hline Persecutory & 57 & 64.8 \\
\hline Reference & 34 & 38.6 \\
\hline Thought alienation & 20 & 22.7 \\
\hline Grandiosity & 17 & 19.3 \\
\hline Hallucinations - types: & Number of Patients & Percentage \% \\
\hline Second person auditory hallucinations & 31 & 46.03 \\
\hline Third person auditory hallucinations & 27 & 40.5 \\
\hline Command hallucination & 9 & 13.4 \\
\hline Tactile hallucinations & 12 & 17.9 \\
\hline Olfactory hallucinations & 4 & 5.97 \\
\hline Diagnosis & Number of patients & Percentage \% \\
\hline Schizophrenia & 51 & 50.5 \\
\hline Manic episode with psychotic features & 13 & 12.9 \\
\hline Delusional disorder & 9 & 8.9 \\
\hline Acute and transient psychotic disorder & 9 & 8.9 \\
\hline Severe depressive episode with psychotic features & 7 & 6.9 \\
\hline
\end{tabular}

\section{Discussion}

This study describes psychopathology seen in a cohort of patients with first episode psychosis. The most prominently featured psychopathology were speech abnormalities, non-euthymic mood, delusions, hallucinations and lack of insight.

Of the delusional themes, delusions of persecution were the most common, with delusions of reference also being featured prominently. This is comparable with previous findings from a dedicated early intervention service i.e., the Early Psychosis Prevention and Intervention Centre (EPPIC), in Melbourne, Australia (9). This suggests that despite differences in geographical location, socio-cultural aspects and the type of service, there is a remarkable similarity in psychopathology in those with FEP. This is further exemplified by the study done by Paolini et al., which reported paranoia to be the most common delusional theme in first episode psychosis (11). However, in a study on first-episode psychosis done by GutierrezLobos et al., jealous, religious, erotomanic and hypochondriacal delusional themes too featured prominently (12). The difference in the age distributions in the two studies may explain this variability, as a majority of the patients in the current study were in a younger age group (13-26 years), whereas in the study by Gutierrez-Lobos et al., most participants were older, with a mean age of 48.3 years. (12). As themes of delusions tend to be related to life problems and experiences, this difference is not unexpected $(5,6)$. 
Of those with hallucinations, second person auditory hallucinations were the most common. This again was comparable to findings of the Australian study (9), which further exemplifies the cross-cultural similarity of presentations. More complex hallucinations such as third person auditory hallucinations were less frequently seen, which can be explained by the early course of the illness (4). Interestingly none of the patients in the current study had reported visual hallucinations, in contrast to studies conducted in Australia and Norway, where visual hallucinations were reported in upto $21 \%$ and $26.5 \%$ of patients with FEP, respectively $(9,13)$. This finding may have been influenced by differences in the proportion of participants who had been diagnosed with drug induced psychosis - in the study done in Melbourne the most common diagnosis was schizophreniform disorder, followed by drug-induced psychosis (9). In contrast, very few participants in our study had received a diagnosis of a drug-induced psychosis.

In our study only $9.9 \%$ of patients reported suicidal ideation. This was markedly different to the study done in Melbourne (9), where almost half of the sample has reported suicidal ideation. This is an interesting difference given the high rates of suicide and deliberate self-harm in Sri Lanka (14).

\section{Limitations}

There were certain limitations in this study, the most significant being the retrospective nature of the study, which necessitated dependence on details recorded in bed head tickets. However, this method has been used in previous first episode psychosis studies as well (9, $12,15)$. The lack of use of a standard assessment tool such as the positive and negative syndrome scale (PANSS) may also have led to omissions (16). Furthermore, negative symptoms were recorded only rarely in the bed head tickets, and thus maybe underreported in our study. The use of several investigators for data extraction may have led to variability in data collection, though all attempts were made to minimise this by use of a structured tool to extract data, use of investigators who had obtained an MD in psychiatry, and provision of workshops to familiarise the data extraction tool.

\section{Conclusions}

Early identification is a challenge in patients with first episode psychosis. Nevertheless, it is of great importance, as a longer duration of psychosis without treatment leads to poor outcomes (3). However, it has been suggested that clinicians are sometimes uncomfortable in exploring psychotic symptoms (17). Therefore, a better understanding of the phenomenology of the FEP is of importance for clinicians, and is likely to help early identification of patients with first episode psychosis, leading to better treatment outcomes. To our knowledge this is the first study done in Sri Lanka exploring psychopathology in patients with FEP, and we anticipate that these findings will help identify patients early in the Sri Lankan setting. Further research is needed, minimising the limitations mentioned above, to improve our understanding of the phenomenology of patients with FEP.

\section{Acknowledgements}

The authors would like to acknowledge the support given by the staff of ward 59, National Hospital, Sri Lanka.

\section{Declaration of interest}

None declared

N S Balasooriya, General Hospital (Teaching) Kandy H G V W Wijesiri, Department of Psychiatry, Faculty of Medicine, University of Peradeniya

R Hanwella, V de Silva, Department of Psychological Medicine, Faculty of Medicine, University of Colombo.

Corresponding author: N S Balasooriya

Email: nilanthab@gmail.com

http://orcid.org/0000-0002-7934-5049

\section{References}

1. Nordentoft M, Jeppesen P, Abel M, et al. OPUS study: suicidal behaviour, suicidal ideation and hopelessness among patients with first-episode psychosis. One-year follow-up of a randomised controlled trial. Br J Pychiatry 2002; 43: s98-106.

2. Malla A, Payne J. First-episode psychosis: psychopathology, quality of life, and functional outcome. Schizophr Bull 2005; 3: 650-71.

3. Simonsen E, Friis S, Haahr U, et al. Clinical epidemiologic first-episode psychosis: 1-year outcome and predictors. Acta Psychiatr Scand 2007; 116(1): 54-61.

4. Hafner H, Maurer K, Loffler W, Riecher-Rossler A. The influence of age and sex on the onset and early course of schizophrenia. Br J Psychiatry 1993; 162: 80-6.

5. Jakes S, Rhodes J, Issa S. Are the themes of delusional beliefs related to the themes of life-problems and goals? Journal of Mental Health 2009; 13(6): 611-9.

6. Raune D, Bebbington P, Dunn G, Kuipers E. Event attributes and the content of psychotic experiences in first-episode psychosis. Psychol Med 2006; 36(2): 221-30.

7. Farooq S, Large M, Nielssen O, Waheed W. The relationship between the duration of untreated psychosis and outcome in low-and-middle income countries: a systematic review and meta analysis. Schizophr Res 2009; 109(1-3): 15-23.

8. Scully PJ, Coakley G, Kinsella A, Waddington JL. Psychopathology, executive (frontal) and general cognitive 
impairment in relation to duration of initially untreated versus subsequently treated psychosis in chronic schizophrenia. Psychol Med 1997; 27(6): 1303-10.

9. Rajapakse T, Garcia-Rosales A, Weerawardene S, Cotton $\mathrm{S}$, Fraser R. Themes of delusions and hallucinations in firstepisode psychosis. Early Interv Psychiatry 2011; 5(3): 254-8.

10. Breitborde NJ, Srihari VH, Woods SW. Review of the operational definition for first-episode psychosis. Early Interv Psychiatry 2009; 3(4): 259-65.

11. Paolini E, Moretti P, Compton MT. Delusions in first-episode psychosis: Principal component analysis of twelve types of delusions and demographic and clinical correlates of resulting domains. Psychiatry Res 2016; 243: 5-13.

12. Gutierrez-Lobos K, Schmid-Siegel B, Bankier B, Walter $H$. Delusions in first-admitted patients: gender, themes and diagnoses. Psychopathology 2001; 34(1): 1-7.

13. Solesvik M, Joa I, Larsen TK, Langeveld J, Johannessen
JO, Bjornestad J, et al. Visual Hallucinations in First-Episode Psychosis: Association with Childhood Trauma. PLoS One 2016; 11(5): e0153458.

14. de Silva VA, Senanayake SM, Dias P, Hanwella R. From pesticides to medicinal drugs: time series analyses of methods of self-harm in Sri Lanka. Bull World Health Organ 2012; 90(1): 40-6.

15. Conus P, Cotton S, Schimmelmann BG, McGorry PD, Lambert M. The First-Episode Psychosis Outcome Study: premorbid and baseline characteristics of an epidemiological cohort of 661 first-episode psychosis patients. Early Intervention in Psychiatry 2007; 1(2): 191-200.

16. Kay SR, Fiszbein A, Opler LA. The positive and negative syndrome scale (PANSS) for schizophrenia. Schizophr Bull 1987; 13(2): 261-76.

17. McCabe R, Heath C, Burns T, Priebe S. Engagement of patients with psychosis in the consultation: conversation analytic study. BMJ 2002; 325(7373): 1148-51. 